"Nexus between financial innovations, remittances and credit performance:

Evidence from augmented ARDL and nonlinear ARDL"

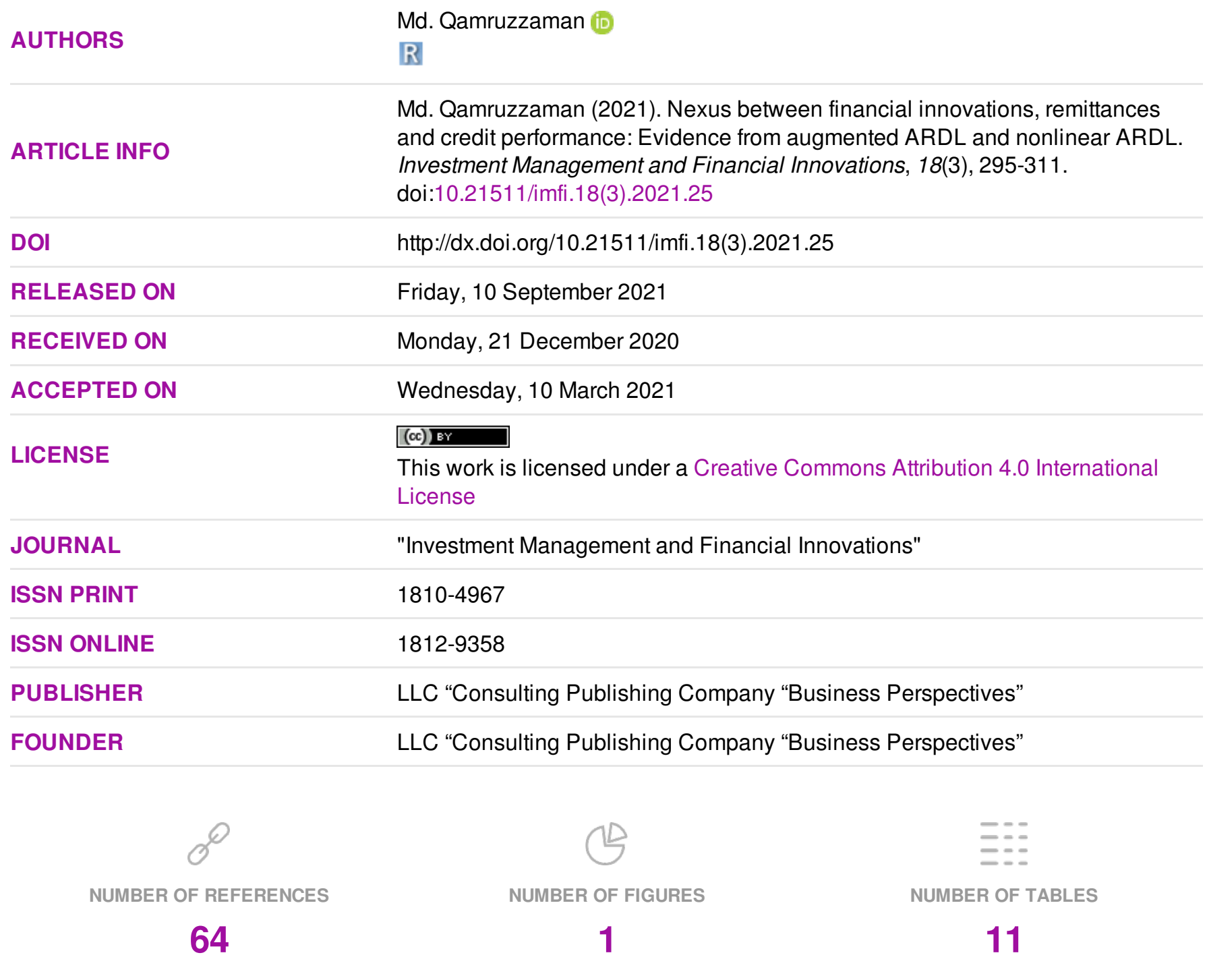

(C) The author(s) 2021. This publication is an open access article. 


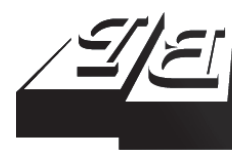

BUSINESS PERSPECTIVES

(0)

LLC "CPC "Business Perspectives" Hryhorii Skovoroda lane, 10, Sumy, 40022, Ukraine www.businessperspectives.org
Received on: $21^{\text {st }}$ of December, 2020 Accepted on: $10^{\text {th }}$ of March, 2021 Published on: $10^{\text {th }}$ of September, 2021

(c) Md. Qamruzzaman, 2021

Md. Qamruzzaman, Associate Professor, School of Business and Economics, United International University, Dhaka, Bangladesh.

Md. Qamruzzaman (Bangladesh)

\title{
NEXUS BETWEEN FINANCIAL INNOVATIONS, REMITTANCES AND CREDIT PERFORMANCE: EVIDENCE FROM AUGMENTED ARDL AND NONLINEAR ARDL
}

\begin{abstract}
The motivation for this study is to assess the impact of financial innovation and remittances on bank-based financial institutions' credit performance in Bangladesh for the period 1981-2019. The study applies augmented ARDL (AARDL) and nonlinear ARDL (NARDL) to identify both long-run and short-run effects and directional causality by performing non-granger casualty tests. AARDL confirms the presence of a long-run association between financial innovation, remittance, trade openness, FDI, and credit performance, which is measured by non-performing loans. In the long run, financial innovation and FDI volatility expose a positive link with NPLs, but remittance inflows and trade openness establish a negative association. Asymmetry shocks in financial innovation reveal a positive relationship with credit performance.

In contrast, the asymmetric shock of remittance and trade openness unveil a negative tie to credit performance, especially in the long run. Furthermore, directional causality provides evidence to support a feedback hypothesis explaining causality between financial innovation and credit performance, as well as remittance inflows and credit performance. These findings suggest that credit performance is guided by future development in remittances and financial innovation; thus, closer attention from policymakers and financial experts is persistent to capitalize or mitigate the impact of the financial system.
\end{abstract}

\section{Keywords}

JEL Classification

\section{INTRODUCTION}

The effect of non-performing loans on financial systems is widely used at an international forum. Financial institutions' performance and financial sustainability are adversely affected by the increase of NPLs in the financial system. Therefore, financial institutions repetitively tried to minimize the conversation rate of NPLs arising from the creation of their assets. Furthermore, over the past decades, several reforms and projects have been carried out aimed at reforming banking and financial structures in numerous countries. The enhanced quality of banks' reserves is due to faster growth in nominal income and credit, expanded financial inclusion, as well as the attempts of supervisory authorities to enhance the control of banks' credit risk and underwriting activities. In recent years, the non-performing loan - fundamental macro nexus have attracted immense interest among scholars (Dimitrios et al., 2016; Anjom \& Karim, 2016; Turan \& Koskija, 2014; Ghosh, 2015; Grigoli et al., 2018; Makri et al., 2014).

Credit mobilization is one way to create assets for financial institutions in the financial system, especially bank-based institutions. 
Credit provision acts as a double-edged sword due to efficient allocation. Credit management allows financial institutions to maximize earnings and credit defaulter, which creates unintended pressure that detrimentally causes operational performances. Thus, credit creation and efficient management for financial institutions emerged as one of the discussant issues among researchers, policymakers, and academicians. Credit defaults, only non-performing loans hereafter NPLSs, established the significant fact responsible for financial volatility and mostly hindered the normal financial development process (Amin et al., 2019).

Financial efficiency is the critical determinant for inclusive and sustainable economic growth (Schumpeter, 1911), since financial inefficiency deteriorates capital accumulation and underpins economic growth. The prime effect observed due to financial inefficiency is the continuous increase of non-performing loans (NPLs) in the financial system. Hence, NPLs induce financial pollution and diminish social benefits (Minsky, 1964; Stiglitz \& Weiss, 1981). An efficient financial system's role is critically addressed in achieving sustainable economic growth by accumulating capital and increasing society's saving propensity. However, the efficiency of the banking sector will be hindered if banks are exposed to speculative loans and financial investments, which, if not adequately controlled, can lead to creating non-performing loans, which can lead to a destabilized financial system (Fofack, 2005) and severe banking crises (Reinhart \& Rogoff, 2011).

This study's prime motivation is to unsheathe the new insight by investigating the effects of financial innovation, remittance inflows, the volatility of FDI, and trade openness on non-performing loans in Bangladesh. To investigate the impact from selected independent variables, namely, trade openness, FDI volatility, remittance, and financial innovation to non-performing loans, this study applies several econometric tools such as ARDL bound test proposed by Pesaran et al. (2001), ARDL t-test proposed by McNown et al. (2018), and augmented ARDL with F-test proposed by Sam et al. (2019). Furthermore, asymmetric relationships were investigated by following nonlinear ARDL proposed by Shin et al. (2014). The directional causality was established by performing the non-granger causality test proposed by Toda and Yamamoto (1995).

The paper is structured as follows: Section 1 contains the survey of related literature and hypotheses development. Section 2 defined variables and explains research methodology. The empirical model estimation and the interpretation are exhibited in Section 3. Finally, the last section provides conclusion.

\section{LITERATURE REVIEW AND HYPOTHESES DEVELOPMENT}

For the last decades, NPLs have become troublesome and one of the most critical issues globally because of banks' profitability and liquidity hamper. Overall economic growth is negatively affected by the increased amount of non-performing loans (Klein, 2013). NPLs respond to macroeconomic, bank-specific, and other global factors. Moreover, wrong customer selection, intense competition, poor management, lack of supporting facilities, poor cash flow, delayed disbursement of funds, and absence of proper monitoring are the gener- al reasons behind the NPLs. Besides, fundamental macro factors such as lower GDP, unemployment rate (Jiménez \& Saurina, 2005), inflation rate, interest rate, and the exchange rate (D. Louzis et al., 2010) can be considered as the root causes of NPLs. Furthermore, bank-specific factors (credit growth, bank size, risk-taking, return on asset, return on equity, solvency, inefficiency, liquidity) and global factors, NPLs arise (Boudriga et al., 2009). In addition, macroeconomic and bank-specific variables affect non-performing loans (Anjom \& Karim, 2016; Md Qamruzzaman et al., 2020). As external economic factors are uncontrollable and affect the overall performance of financial institutions, compared to the bank-specific factors, NPLs react most by the macroeconomic factors (Kjosevski 
\& Petkovski, 2017; Klein, 2013; D. P. Louzis, et al., 2012; Polodoo et al., 2015), while opposite conclusions were made by Amuakwa-Mensah and Boakye-Adjei (2015), Dimitrios et al. (2016), Haniifah (2015), and Rifat (2016).

In European countries, NPLs are found to increase when unemployment rises, the exchange rate depreciates, and inflation is high (Klein, 2013; D. P. Louzis et al., 2012; Škarica, 2014; Turan \& Koskija, 2014). However, opposite findings are found in Shingjergji (2013). An insignificant relationship has been found between GDP, interest rate, inflation, exchange rate, and NPLs (Haniifah, 2015). In a general sense, GDP and NPLs should be strongly related because higher GDP mostly expresses a country's robust economic condition. Non-performing loans cannot exist too much in countries with healthy economic conditions (Ghosh, 2015; Rajha, 2016). On the other hand, a positive relationship has been found between GDP and NPLs (Anjom \& Karim, 2016; Poudel, 2013; Shingjergji, 2013). Moreover, banks' profitability also influences NPLs to some extent. Banks that are profitable and have lower exposure to the household sectors tend to have lower NPLs (Beaton \& Myrvoda, 2016; Isik \& Bolat, 2016).

Money supply (broad money) is also a strong influencer of NPL, but its impact is ambiguous as it can create both positive and negative impacts on NPL. Thus, though money supply is used in a few papers as independent variables, different results are found in Morakinyo and Sibanda (2016) and Poudel (2013). Because of rising domestic credit to the private sector (loans, purchases of securities, trade credits, account receivables), there is a probability of increasing NPL. So, there must be a positive impact of credit to the private sector on NPL (Akinlo \& Emmanuel, 2014; Beaton \& Myrvoda, 2016; Dorina \& Colesnicova, 2014; Kjosevski \& Petkovski, 2017). On the other hand, NPL are used to respond negatively with remittances, as the remittance inflow keeps the general people from taking excessive loans from banks, and a decrease in loans tends to decrease NPLs. Thus, NPL is negatively related to remittances (Dorina \& Colesnicova, 2014; Turan \& Koskija, 2014).

Kumar et al. (2018) investigated the NPLs' determents of the banking industry in Fiji for the peri- od 2000-2013. They found that macro fundaments, such as economic growth, exchange rate, inflation, and unemployment, attribute to the buildup of NPLs. On the other hand, remittance inflows create downward pressure on NPLs pileup during the research period. Furthermore, Adebola et al. (2011) investigated the key factors responsible for expanding NPLs in Malaysia's Islamic banking sector for 2007M1-2009M12. They concluded that interest rate and industrial production index exhibited a positive association for an increase of NPLs of Islamic banking in Malaysia in the long run. However, the producer price index appears with an antagonistic relationship both in the long and short run.

Rahman and Jahan focused on NPLs determinants for Islamic banking with firms' specifics factors. Study findings revealed that firms' profitability attributes to increasing non-performing loans. They argued that asset creation through sectioning excess credit offered higher interest earnings and created the possibility of augmenting NPLs. Panel data from 16 banks for the period 1990-2005 were used.

This study is not aimed at a comprehensive investigation to identify the critical determinants for the increase in NPLs in Bangladesh. Instead, it explores the nexus between Financial Innovation, Remittance Inflows, Trade Openness, FDI Volatility and NPLs for the first time by applying both linear frameworks that are bound testing proposed by Pesaran et al. (2001), $t$-test proposed by McNown et al. (2018), augmented ARDL proposed by Sam et al. (2019), and nonlinear framework, that is nonlinear ARDL, proposed by Shin et al. (2014). The study tests six hypotheses pertinent to the conceptual model of the study. Figure 1 represents the possible causality between financial inclusion, stock market development, and foreign capital from a theoretical perspective.

\section{METHODS}

The study utilizes annualized time series data for the period 1981-2019. As a dependent variable, non-performing loans are measured by the ratio of NPLs to gross domestic product (Chen et al., 2018). Because of broad regional economic growth 


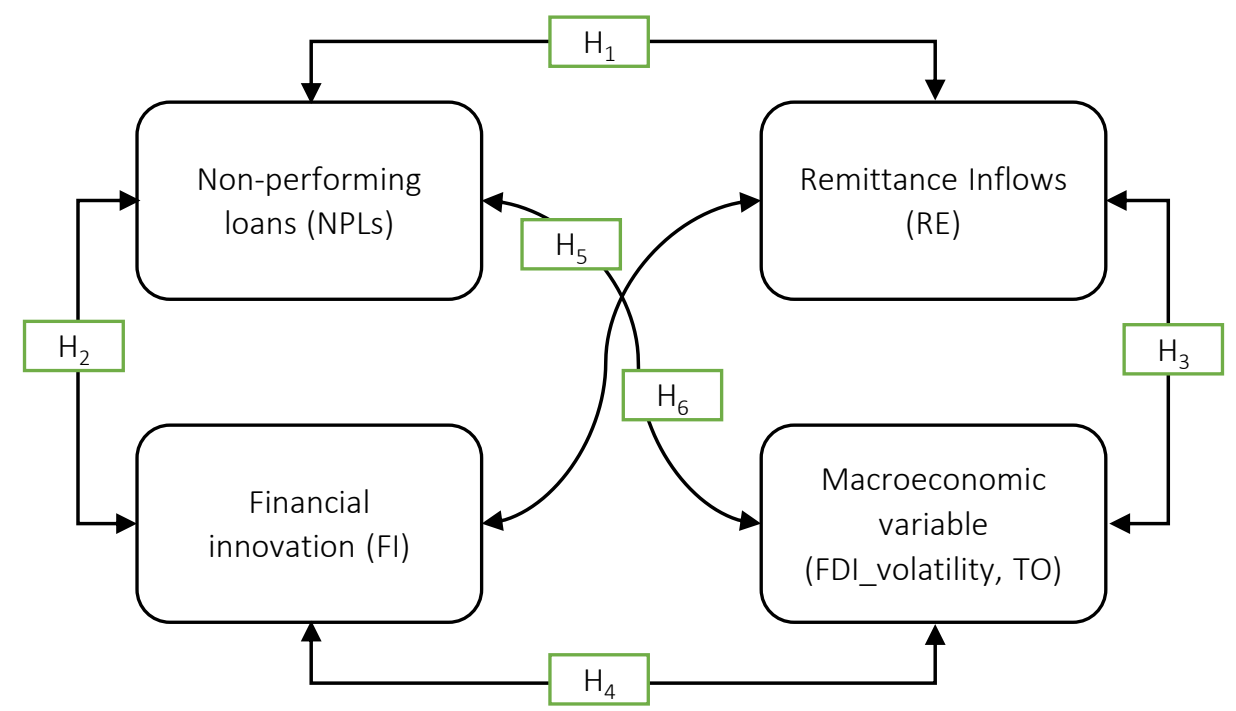

Note: $H_{1 A}$ : Remittance inflows cause non-performing loans, and vice versa; $H_{2 A}$ : Financial innovation causes non-performing loans, and vice versa; $H_{3 A, B}$ : Remittance inflows cause a macroeconomic variable, and vice versa; $H_{4 A, B}$ : Financial innovation causes a macroeconomic variable, and vice versa; $H_{5 A, B}$ : Stock market development causes financial inclusion, and vice versa; and $H_{6 A, B}$ : Non-performing loans cause a macroeconomic variable, and vice versa.

Figure 1. Conceptual model for hypotheses testing

disparities, non-performing loans of commercial banks can be affected by regional economic efficiency. Therefore, the non-performing credit ratio is preferred to calculate non-performing commercial bank loans to avoid economic growth. Remittances are measured by the per capita remittance received: Personal remittances comprise personal transfers and compensation of employees. Personal transfers consist of all current transfers in cash or in-kind made or received by residents. It is expected that continuous inflows of remittances in the economy can reduce the trend of increasing non-performing loans in the economy. The expected sign of remittance $(R)$ is negative. As a proxy for financial innovation, empirical literature suggests three indicators widely used such as the ratio of M2 to M1 (Md Qamruzzaman \& Karim, 2020a), the ratio of M3/M2 (Md. Qamruzzaman \& Wei Jianguo, 2018; Md Qamruzzaman \& Wei, 2019), and the growth of domestic credits to the private sector (Md. Qamruzzaman \& Jianguo, 2017; Md Qamruzzaman \& Wei, 2018). Addressing the effects on NPLs from a different proxy of financial innovation, this study selects all three for enhancing the robustness of empirical model estimation. Trade openness (TO) is measured by the sum of import and export as a percentage of GDP. And finally, the FDI volatility is measured by performing ARCH effects of annualized data.
Table 1. Variable definition and sources

\begin{tabular}{|c|c|c|c|}
\hline Variables & Notation & Definition & Data sources \\
\hline $\begin{array}{l}\text { Non- } \\
\text { performing } \\
\text { loans }\end{array}$ & $N P L s$ & $\begin{array}{c}\text { NPLs as a } \\
\text { percentage of GDP }\end{array}$ & $\begin{array}{c}\text { Bangladesh } \\
\text { Bank Annual } \\
\text { reports } \\
\end{array}$ \\
\hline \multicolumn{4}{|c|}{ Financial innovation } \\
\hline M2/M1 & $\mathrm{FI}_{1}$ & The ratio of $\mathrm{M} 2 / \mathrm{M}$ & $\begin{array}{c}\text { Author } \\
\text { calculation }\end{array}$ \\
\hline M3/M2 & $\mathrm{FI}_{2}$ & $\begin{array}{l}\text { The ratio of Broad } \\
\text { money to Narrow }\end{array}$ & \\
\hline DCP & $\mathrm{FI}_{3}$ & $\begin{array}{l}\text { Domestic credit } \\
\text { private section as a } \\
\text { percentage of GDP }\end{array}$ & WDI \\
\hline $\begin{array}{l}\text { Trade } \\
\text { openness }\end{array}$ & TO & $\begin{array}{l}\text { As a ratio of total } \\
\text { trade to GDP }\end{array}$ & WDI \\
\hline Remittance & $\mathrm{R}$ & $\begin{array}{l}\text { Per capital } \\
\text { remittance received }\end{array}$ & WDI \\
\hline $\begin{array}{l}\text { FDI } \\
\text { volatility }\end{array}$ & $\mathrm{FDI}_{\text {vol }}$ & $\begin{array}{l}\text { ARCH effects of } \\
\text { FDI inflows in the } \\
\text { economy }\end{array}$ & $\begin{array}{l}\text { Authors } \\
\text { calculation }\end{array}$ \\
\hline
\end{tabular}

The generalized conceptual empirical model can be established as follows:

$$
N P L=\int \text { Remitane, Financial innovation, }
$$

Trade Opennes, FDI $I_{\text {volatility }}$.

Transforming equation (1) into a linear form can be represented as follows:

$$
\begin{aligned}
& \ln N P L_{t}=\alpha_{0}+\beta_{1} \ln R E_{t}+\beta_{2} \ln F I_{t}+ \\
& +\beta_{3} \ln T O_{t}+\beta_{3} \ln F D I_{-} v_{t}+\varepsilon_{t},
\end{aligned}
$$


where $R E$ is the exchange rate, FI stands for financial innovation, FDI_volatility represents variability in foreign direct investment, TO represents trade openness, and $G$ is the real government expenditure. Model coefficients of $\beta_{1}$ to $\beta_{3}$ in equation (2) represent long-run elasticities, and $\varepsilon_{t}$ stands for error correction term the equations.

\subsection{Autoregressive distributed lag (ARDL)}

In recent times, when investigating the long-run association, empirical studies extensively use a framework proposed by Pesaran, known as ARDL (Md Qamruzzaman \& Wei Jianguo, 2018; Md Qamruzzaman et al., 2020; Md Qamruzzaman \& Karim, 2020a, 2020b). ARDL estimation possesses certain benefits over traditional cointegration tests, including (1) efficient estimation regardless of the study's sample size (Ghatak \& Siddiki, 2001), (2) ability of handling mixed-order variable integration, and model stability and efficiency can be obtained by selecting appropriate lagged specification (Pesaran et al., 2001), (3) unbiased estimation for both long-run and short-run elasticity (Banerjee et al., 1993).

Following Pesaran et al. (2001), the generalized ADRL model for the study with different proxies for NPLs is as follows:

$$
\begin{aligned}
& \Delta \ln N P L_{t}=\alpha_{0}+\sum_{i=1}^{n} \mu_{1} \Delta \ln N P L_{t-i}+ \\
& +\sum_{i=0}^{n} \mu_{2} \Delta \ln R E_{t-i}+\sum_{i=0}^{n} \mu_{3} \Delta \ln F I_{t-i}^{*}+ \\
& +\sum_{i=0}^{n} \mu_{6} \Delta \ln T O_{t}+ \\
& +\sum_{i=0}^{n} \mu_{7} \Delta \ln F D I_{-} \text {volatility }_{t-i}+ \\
& +\gamma_{1} \ln N P L_{t-1}+\gamma_{2} \ln R E_{t-1}+ \\
& +\gamma_{3} \ln F I_{t-1}^{1}+\gamma_{6} \ln T O_{t-1}+ \\
& +\gamma_{6} \ln F D I_{-} \text {volatility }_{t-1}+\omega_{1 t} .
\end{aligned}
$$

where $\Delta$ indicates differencing of variables, while is the error term (white noise), and ( $t-1)$ is for the lagged period, is the long-run coefficient. Based on linear ARDL equations 3-5, long-run coefficient should be available from $\gamma_{1}$ to $\gamma_{5}$ and short- run coefficients should be obtained from $\mu_{1}$ to $\mu_{5}$ from each empirical model estimation. Long-run association between variables should be tested following the F-test (Pesaran et al., 2001) and the

\begin{tabular}{|c|c|c|}
\hline $\begin{array}{c}\text { Cointegration } \\
\text { test }\end{array}$ & Null hypothesis & $\begin{array}{l}\text { Alternative } \\
\text { hypothesis }\end{array}$ \\
\hline$F$-bound test & $\begin{array}{l}\gamma_{1}=\gamma_{2}=\gamma_{3}= \\
=\gamma_{4}=\gamma_{5}=\gamma_{6}= \\
=\gamma_{7}=0\end{array}$ & $\begin{array}{c}\text { Any, } \\
\gamma_{1}, \gamma_{2}, \gamma_{3}, \gamma_{4}, \\
\gamma_{5}, \gamma_{6}, \gamma_{7} \neq 0\end{array}$ \\
\hline $\begin{array}{l}t \text {-test on } \\
\text { a lagged } \\
\text { dependent } \\
\text { variable }\end{array}$ & $\gamma_{1}=0$ & $\gamma_{1} \neq 0$ \\
\hline $\begin{array}{l}\text { F-test on } \\
\text { a lagged } \\
\text { independent } \\
\text { variable }\end{array}$ & $\begin{array}{l}\gamma_{2}=\gamma_{3}=\gamma_{4}=\gamma_{5}= \\
=\gamma_{6}=\gamma_{7}=0\end{array}$ & $\begin{array}{c}\text { Any, } \\
\gamma_{2}, \gamma_{3}, \gamma_{4}, \\
\gamma_{5}, \gamma_{6}, \gamma_{7} \neq 0\end{array}$ \\
\hline
\end{tabular}
t-test on the lagged level of the dependent variable as suggested by Pesaran et al. (2001), as well as another additional F-test on the lagged levels of the independent variable(s) as suggested by McNown et al. (2018).

Table 2. Null hypotheses for all three tests

Recently, investigating the asymmetry association between dependent and independent variables, non-linear ARDL emerged as a pioneering framework in empirical studies (Md Qamruzzaman et al., 2020; Md Qamruzzaman et al., 2019), which is proposed by Shin et al. (2014). Investigation of non-linear effects from independent variables to dependent variables requires integrating variables decomposition into positive and negative shocks. The decomposition of positive and negative shocks of explanatory variables can be computed by using the following equations:

$$
\left\{\begin{array}{l}
\operatorname{POS}\left(F D I_{v o l}\right)_{t}=\sum_{k=1}^{t} \ln F D I_{v o l_{k}^{+}}= \\
=\sum_{k=1}^{T} \operatorname{MAX}\left(\Delta \ln F D I_{v o l_{k}}, 0\right) \\
N E G\left(F D I_{v o l}\right)_{t}=\sum_{k=1}^{t} \ln F D I_{v o l_{k}^{-}}= \\
=\sum_{k=1}^{T} \operatorname{MIN}\left(\Delta \ln F D I_{v o l_{k}}, 0\right)
\end{array}\right.
$$




$$
\begin{aligned}
& \left\{\begin{array}{l}
P O S(T O)_{t}=\sum_{k=1}^{t} \ln T O_{k}^{+}=\sum_{k=1}^{T} M A X\left(\Delta \ln T O_{k}, 0\right) \\
N E G(T O)_{t}=\sum_{k=1}^{t} \ln T O_{k}^{-}=\sum_{k=1}^{T} M I N\left(\Delta \ln T O_{k}, 0\right)
\end{array}\right. \\
& \left\{\begin{array}{l}
P O S(R)_{t}=\sum_{k=1}^{t} \ln R_{k}^{+}=\sum_{k=1}^{T} M A X\left(\Delta \ln R_{k}, 0\right) \\
N E G(R)_{t}=\sum_{k=1}^{t} \ln R_{k}^{-}=\sum_{k=1}^{T} M I N\left(\Delta \ln R_{k}, 0\right)
\end{array}\right. \\
& \left\{\begin{array}{l}
P O S\left(F I^{*}\right)_{t}=\sum_{k=1}^{t} \ln F I^{*}{ }_{k}^{*}=\sum_{k=1}^{T} M A X\left(\Delta \ln F I^{1}{ }_{k}, 0\right) \\
N E G\left(F I^{*}\right)_{t}=\sum_{k=1}^{t} \ln F I^{*-}{ }_{k}=\sum_{k=1}^{T} M I N\left(\Delta \ln F I^{1}, 0\right)
\end{array} .\right.
\end{aligned}
$$

Now the asymmetric shocks derived from equations 4-7 are incorporated in equation (3) and reproduced in a nonlinear framework, which is as follows:

$$
\begin{aligned}
& \Delta \ln N P L_{t, i}=\alpha_{0}+\sum_{i=1}^{n} \mu_{1} \Delta N P L_{t-i}+ \\
& +\sum_{i=0}^{m}\left(\mu_{2}^{+} \Delta F D I_{v o l_{t-i}}+\mu_{2}^{-} \Delta F D I_{v o l_{t-i}}\right)+ \\
& +\sum_{i=0}^{r}\left(\mu_{3}^{+} \Delta T O_{t-i}+\mu_{3}^{-} \Delta T O_{t-i}\right)+ \\
& +\sum_{i=0}^{r}\left(\mu_{4}^{+} \Delta F I_{t-i}+\mu_{4}^{-} \Delta F I_{t-i}\right)+ \\
& +\sum_{i=0}^{r}\left(\mu_{4}^{+} \Delta R_{t-i}+\mu_{4}^{-} \Delta R_{t-i}\right)+ \\
& +\gamma_{0} N P L_{t-1}+\left(\gamma_{1}^{+} F D I_{v o l_{t-1}}+\gamma_{1}^{-} F D I_{v o l_{t-1}}\right)+ \\
& +\left(\gamma_{2}^{+} T O_{t-1}+\gamma_{2}^{-} T O_{t-1}\right)+ \\
& +\left(\gamma_{3}^{+} F I_{t-1}+\gamma_{3}^{-} F I_{t-1}\right)+\left(\gamma_{4}^{+} R_{t-1}+\gamma_{4}^{-} R_{t-1}\right)+\omega_{t} .
\end{aligned}
$$

To investigate the existence of the long-run asymmetric relationship, Shin et al. (2014) proposed a bound test, which is a joint test of all lagged levels of regressors. Wald F-test is utilized to test the null hypothesis that thereis no asymmetric relationship $H_{0}$ : $\gamma_{0}=\gamma_{1}^{+}=\gamma_{1}^{-}=\gamma_{2}^{+}=\gamma_{2}^{-}=\gamma_{3}^{+}=\gamma_{3}^{-}=\gamma_{4}^{+}=\gamma_{4}^{-}=0$, against the alternative hypothesis $H_{1}$ : $\gamma_{0} \neq \gamma_{1}^{+} \neq \gamma_{1}^{-} \neq \gamma_{2}^{+} \neq \gamma_{2}^{-} \neq \gamma_{3}^{+} \neq \gamma_{3}^{-} \neq \gamma_{4}^{+} \neq \gamma_{4}^{-} \neq 0$. The rejection of the null hypothesis establishes an asymmetric relationship available in the empirical equation.
Non-linear ARDL model is evaluated under three assessments. First, the short-run asymmetry, that is $\mu_{1}^{+} \neq \mu_{1}^{-}$for FDI volatility, $\mu_{2}^{+} \neq \mu_{2}^{-}$for trade openness; $\mu_{3}^{+} \neq \mu_{3}^{-}$for financial innovation, and $\mu_{4}^{+} \neq \mu_{4}^{-}$for remittance inflows in the equation. Second, short-run asymmetry impact confirmed, if $\sum \mu_{1}^{+} \neq \sum \mu_{1}^{-}$for FDI volatility; $\sum \mu_{3}^{+} \neq \sum \mu_{3}^{-}$ for trade openness; $\sum \mu_{4}^{+} \neq \sum \mu_{4}^{-}$for financial innovation; and $\sum \mu_{5}^{+} \neq \sum \mu_{5}^{-}$for remittance inflows, respectively. Third, long-run asymmetry is ascertained if $\gamma_{1}^{+} \neq \gamma_{1}^{-}$for the FDI volatility, $\gamma_{2}^{+} \neq \gamma_{2}^{-}$for the trade openness, $\gamma_{3}^{+} \neq \gamma_{3}^{-}$for financial innovation, and $\gamma_{4}^{+} \neq \gamma_{4}^{-}$for remittance inflows.

To establish directional causality between financial innovation, money supply, interest rate, remittances, and stock price, the non-causality test proposed by Toda and Yamamoto (1995) was applied. The Toda and Yamamoto (1995) causality test is based on the idea of Vector autoregression at level $\left(P=K+D_{\max }\right)$ with correct VAR order $K$ and $d$ extra lag, where $d$ represents the maximum order of integration of time series. The following causal equation is used to detect possible directional association between variables:

$$
\begin{aligned}
& N P L_{t(p c b)}=\alpha_{0}+\sum_{i=1}^{k} \beta_{1 i} N P L_{t-i}+ \\
& +\sum_{j=k+1}^{d_{\text {max }}} \beta_{2 j} N P L_{t-j}+\sum_{i=1}^{k} \gamma_{1 i} R_{t-i}+ \\
& +\sum_{j=k+1}^{d_{\max }} \gamma_{1 j} R_{t-j}+\sum_{i=1}^{k} \pi_{1 i} F I_{t-i}^{1}+ \\
& +\sum_{j=k+1}^{d_{\max }} \pi_{1 j} F I_{t-j}^{1}+\sum_{i=1}^{k} \tau_{1 i} F I_{t-i}^{2}+ \\
& +\sum_{j=k+1}^{d_{\text {max }}} \tau_{1 j} F I_{t-j}^{2}+\sum_{i=1}^{k} \varphi_{1 i} F I_{t-i}^{3}+ \\
& +\sum_{j=k+1}^{d_{\max }} \varphi_{1 j} F I_{t-j}^{3}+\sum_{i=1}^{k} \delta_{1 i} F D I_{v o l_{t-i}}+ \\
& +\sum_{j=k+1}^{d_{\max }} \delta_{2 j} F D I_{v o l_{t-j}}+\sum_{i=1}^{k} \theta_{1 i} T O_{t-i}+ \\
& +\sum_{j=k+1}^{d_{\max }} \theta_{2 j} T O_{t-j}+\varepsilon_{1 t} \cdot
\end{aligned}
$$




\section{RESULTS}

According to the existing literature, empirical estimation using time series data has helped to determine the order of variable integration. In line with literature, the study performs a stationary test by applying the ADF test proposed by Dickey and Fuller (1979), P-P test proposed by Phillips and Perron (1988), KPSS test proposed by Kwiatkowski et al. (1992). The results of unit root tests are displayed in Table 3. The study findings establish a mixed order of integration, indicating that variables are either stationary at a level $\mathrm{I}(0)$ or after the first difference I(1); no variables expose stationarity after the second difference I(2).

Furthermore, the study applies the $\mathrm{Ng}$ and Perron (2001) test, and the right size and power properties are employed. Unit root test under $\mathrm{Ng}$ produces four test statistics, namely MZa, MZt, MSB, and MPT, for investigating the existence or unit roots.
The coefficients $\mathrm{MZ}_{\mathrm{a}}$ and $\mathrm{MZ}_{\mathrm{t}}$ were obtained by modifying Phillips (1987) and Phillips and Perron (1988) $\mathrm{Z}_{\mathrm{a}}$ and $\mathrm{Z}_{\mathrm{t}}$ test. The MSB is extracted from the Bhargava (1986) R test, and lastly, the value of MPT is adopted from Elliott, Rothenberg, and Stock's (1996) point optimal test. Results of the stationary test are exhibited in Table 4.

The results of the long-run cointegration test are reported in Table 5. The study performs nine cointegration equations to ascertain possible long-run association based on NPL measures in the equation and proxy of financial innovation. Because the test statistics of $\mathrm{F}_{\text {overall }}, \mathrm{t}_{\mathrm{DV}}$, and $\mathrm{F}_{\mathrm{IDV}}$ are statistically significant at a $1 \%$ level of significance, these findings suggest the presence of long-run cointegration between financial innovation, remittance, trade openness, FDI volatility, and non-performing loans.

Since the cointegration test of AARDL confirms long-run cointegration in the empiri-

Table 3. Unit root test results

\begin{tabular}{|c|c|c|c|c|c|c|}
\hline & \multicolumn{3}{|c|}{ At level } & \multicolumn{3}{|c|}{ At first difference } \\
\hline & ADF & PP & KPSS & ADF & PP & KPSS \\
\hline$N P L_{p c b s}$ & -1.481 & 0.530 & $0.329_{* * *}$ & $-6.105_{* * *}$ & $-6.109_{* * *}$ & 0.095 \\
\hline$N P L_{s c b s}$ & -1.705 & -1.805 & $0.447_{* * *}$ & $-5.263_{* * *}$ & $-5.272_{* * *}$ & 0.099 \\
\hline$N P L_{f c b s}$ & -1.518 & -1.959 & $0.412_{* * *}$ & $-4.399_{* * *}$ & $-8.392_{* * *}$ & 0.108 \\
\hline$F I^{1}$ & $-9.560 * * *$ & -1.560 & $0.563_{* * *}$ & $-5.978_{* * *}$ & $-8.074_{* * *}$ & 0.081 \\
\hline$F I^{2}$ & $-3.980 * *$ & -3.303 & $0.445_{* * *}$ & $-11.817_{* * *}$ & $-3.577_{* * *}$ & 0.136 \\
\hline$F I^{3}$ & $-18.392^{* * *}$ & -8.249 & $0.430_{* * *}$ & $-5.942_{* * *}$ & $-7.184_{* * *}$ & $0.500_{* * *}$ \\
\hline$F D I_{\text {volatility }}$ & -1.260 & -2.159 & $0.497_{* * *}$ & $-7.997_{* * *}$ & $-3.638_{* *}$ & 0.220 \\
\hline$R E$ & -2.293 & -1.325 & $0.647_{* * *}$ & $-3.240_{* *}$ & $-6.461_{* * *}$ & 0.111 \\
\hline TO & -0.888 & -0.839 & $0.631_{* * *}$ & $-3.794_{* *}$ & $-7.856_{* * *}$ & $0.243_{* *}$ \\
\hline
\end{tabular}

Table 4. Ng-Parron unit root test

\begin{tabular}{|c|c|c|c|c|c|c|c|c|}
\hline & \multicolumn{4}{|c|}{ At level } & \multicolumn{4}{|c|}{ At first difference } \\
\hline & $\mathrm{MZa}$ & MZt & MSB & MPT & $\mathrm{MZa}$ & $\mathrm{MZt}$ & MSB & MPT \\
\hline$N P L_{p c b s}$ & -4.895 & -1.537 & 0.314 & 18.45 & $-16.355^{* *}$ & -2.857 & 0.174 & 5.585 \\
\hline$N P L_{s c b s}$ & $-18.069 * *$ & -2.800 & $0.155^{* *}$ & 6.234 & $-18.505^{* *}$ & -0.790 & 0.427 & 37.822 \\
\hline$N P L_{f c b s}$ & -3.244 & -1.219 & 0.375 & 26.926 & $-16.431^{*}$ & -2.863 & 0.174 & 5.563 \\
\hline$F I^{1}$ & -8.739 & -2.038 & 0.233 & 10.60 & $-16.398^{*}$ & -2.853 & 0.174 & 5.613 \\
\hline$F I^{2}$ & -5.639 & -1.628 & 0.288 & 16.035 & $-16.356^{*}$ & -2.858 & 0.174 & 5.579 \\
\hline$F I^{3}$ & -13.389 & -2.586 & 0.193 & 6.810 & $-14.605^{*}$ & -2.667 & 0.182 & 6.441 \\
\hline$F D I_{\text {volatility }}$ & -4.207 & -1.264 & 0.300 & 19.845 & $-70.773^{*}$ & -5.905 & 0.083 & 1.474 \\
\hline $\mathrm{RE}$ & -7.272 & -1.860 & 0.255 & 12.604 & -16.434 & -2.860 & 0.174 & 5.581 \\
\hline TO & -9.94 & -2.092 & 0.21051 & 9.75269 & -16.115 & -2.799 & 0.173 & 5.886 \\
\hline \multirow{3}{*}{\multicolumn{4}{|c|}{ Asymptotic critical values: Ng and Perron (2001), Table 1}} & $1 \%$ & -23.80 & -3.420 & 0.143 & 4.030 \\
\hline & & & & $5 \%$ & -17.300 & -2.910 & 0.168 & 5.480 \\
\hline & & & & $10 \%$ & -14.200 & -2.620 & 0.185 & 6.670 \\
\hline
\end{tabular}


Table 5. Augmented ARDL cointegration test

\begin{tabular}{|c|c|c|c|c|c|}
\hline & & F-bound & $t$-DV & F-IDV & Remark \\
\hline \multicolumn{6}{|c|}{ Panel A: For private commercial banks } \\
\hline \multicolumn{2}{|l|}{$F\left(N P L_{P C B S}\right)=\left(N P L_{P C B S} / F I_{1}, R O, T O, F D I_{\text {volatility }}\right)$} & 7.211 & -5.646 & 4.988 & Presence \\
\hline \multicolumn{2}{|l|}{$F\left(N P L_{P C B S}\right)=\left(N P L_{P C B S} / F I_{2}, R O, T O, F D I_{\text {volatility }}\right)$} & 11.972 & -8.441 & 10.671 & Presence \\
\hline \multicolumn{2}{|l|}{$F\left(N P L_{P C B S}\right)=\left(N P L_{P C B S} / F I_{3}, R O, T O, F D I_{\text {volatility }}\right)$} & 10.723 & -3.969 & 16.284 & Presence \\
\hline \multicolumn{6}{|c|}{ Panel B: For state-owned commercial banks } \\
\hline \multicolumn{2}{|l|}{$F\left(N P L_{S C B S}\right)=\left(N P L_{S C B S} / F I_{1}, R O, T O, F D I_{v o l}\right)$} & 5.501 & -3.501 & 3.833 & Presence \\
\hline \multicolumn{2}{|l|}{$F\left(N P L_{S C B S}\right)=\left(N P L_{S C B S} / F I_{2}, R O, T O, F D I_{v o l}\right)$} & 7.722 & -3.468 & 4.643 & Presence \\
\hline \multicolumn{2}{|l|}{$F\left(N P L_{S C B S}\right)=\left(N P L_{S C B S} / F I_{3}, R O, T O, F D I_{v o l}\right)$} & 9.552 & -2.921 & 9.808 & Presence \\
\hline \multicolumn{6}{|c|}{ Panel C: For foreign commercial banks } \\
\hline \multicolumn{2}{|l|}{$F\left(N P L_{F C B S}\right)=\left(N P L_{F C B S} / F I_{1}, R O, T O, F D I_{v o l}\right)$} & 6.975 & -4.251 & 13.285 & Presence \\
\hline \multicolumn{2}{|l|}{$F\left(N P L_{F C B S}\right)=\left(N P L_{F C B S} / F I_{2}, R O, T O, F D I_{v o l}\right)$} & 13.574 & -11.125 & 9.848 & Presence \\
\hline \multicolumn{2}{|l|}{$F\left(N P L_{F C B S}\right)=\left(N P L_{F C B S} / F I_{3}, R O, T O, F D I_{v o l}\right)$} & 12.162 & -5.214 & 12.094 & Presence \\
\hline \multirow{2}{*}{\multicolumn{2}{|c|}{ Critical value : $K=4$}} & \multicolumn{2}{|c|}{$5 \%$} & \multicolumn{2}{|c|}{$10 \%$} \\
\hline & $\mathrm{I}(1)$ & $\mathrm{I}(0)$ & $\mathrm{I}(1)$ & $\mathrm{I}(0)$ & $\mathrm{I}(1)$ \\
\hline Pesaran et al. (2001) & 6.368 & 3.276 & 4.63 & 2.696 & 3.898 \\
\hline Narayan t test & -4.6 & -2.86 & -3.99 & -2.57 & -3.66 \\
\hline Sam et al. (2019) & 5.91 & 2.46 & 4.18 & 2.00 & 3.47 \\
\hline
\end{tabular}

cal equation, the next step is to investigate the The following section investigates asymmetric efmagnitude of long-run and short-run coeffi- fects of financial innovation, remittance, trade cients running from financial innovation, FDI openness, and FDI volatility on NPLs by performvolatility, remittance, trade openness and NPLs ing a nonlinear ARDL equation. Table 7 exhibits in Bangladesh. The empirical model results are the results of nonlinear models consisting of a reported in Table 6.

six-panel representation.

Table 6. Long-run and short-run model coefficients

\begin{tabular}{|c|c|c|c|c|c|c|c|c|c|}
\hline & [1] & {$[2]$} & [3] & [4] & [5] & [6] & [7] & {$[8]$} & [9] \\
\hline \multicolumn{10}{|c|}{ Panel A: Long-run coefficients } \\
\hline$F I^{1}$ & $0.063^{* * *}$ & - & - & $0.085^{* * *}$ & - & - & $0.060 * * *$ & - & - \\
\hline$F I^{2}$ & - & $0.070^{*} * *$ & - & - & $0.077^{* * *}$ & - & - & $0.035^{* * *}$ & - \\
\hline$F I^{3}$ & - & - & $0.041^{* * *}$ & - & - & $0.078^{* * *}$ & - & - & $0.022 * * *$ \\
\hline$F D I_{v o l}$ & $0.096 * *$ & $0.084^{* *}$ & $0.035 * *$ & $0.030 * *$ & $0.109 * *$ & $0.034 * * *$ & $0.072 * * *$ & $0.047 * * *$ & $0.087^{* * *}$ \\
\hline$R E$ & $-0.283^{* * *}$ & $-0.257 * * *$ & $-0.161^{* *}$ & $-0.137 * * *$ & $-0.108^{* *}$ & $-0.136^{* * *}$ & $-0.036^{* * *}$ & $-0.099 * * *$ & $-0.069 * * *$ \\
\hline$T O$ & $-0.086^{* * *}$ & $-0.090 * *$ & $-0.066 * * *$ & $-0.194 * *$ & $-0.112 * * *$ & $-0.069 * * *$ & $-0.105^{* * *}$ & $-0.080 * * *$ & $-0.102 * * *$ \\
\hline$C$ & $-10.506^{* * *}$ & $-0.533^{* *}$ & $-9.522^{* * *}$ & $-0.782 * * *$ & $-0.441^{* * *}$ & $-0.362^{* * *}$ & $0.744^{* * *}$ & $-1.102 * * *$ & $-1.445^{* * *}$ \\
\hline \multicolumn{10}{|c|}{ Panel B: Short-run coefficients } \\
\hline$\varphi$ & $-0.389 * * *$ & $-0.655^{* * *}$ & $-0.587 * * *$ & $-0.530 * * *$ & $-0.752 * * *$ & $-0.461^{* * *}$ & $-0.181^{* * *}$ & $-0.174 * * *$ & $-0.183^{* * *}$ \\
\hline$\beta^{1}$ & $0.081^{* * *}$ & & & $0.082 * *$ & & & $0.045^{* *}$ & & \\
\hline$\beta^{2}$ & & $0.085^{* *}$ & & & $-0.040 *$ & & & 0.063 & \\
\hline$\beta^{3}$ & & & $0.012 *$ & & & 0.021 & & & $0.087^{* *}$ \\
\hline$\Upsilon$ & 0.096 & $-0.062 * *$ & $-0.012 * * *$ & $-0.067 * *$ & $-0.098 * *$ & $-0.016^{*}$ & $-0.076^{*}$ & $-0.017 * *$ & $-0.094 * *$ \\
\hline$\mu$ & $-0.081^{* * *}$ & $-0.019 * * *$ & $-0.012^{* *}$ & $-0.026^{* * *}$ & $-0.006^{* * *}$ & $-0.049 * * *$ & $-0.068^{* *}$ & $-0.053^{* *}$ & $-0.025^{* *}$ \\
\hline
\end{tabular}


Table 6 (cont.). Long-run and short-run model coefficients

\begin{tabular}{|c|c|c|c|c|c|c|c|c|c|}
\hline & [1] & [2] & [3] & [4] & [5] & [6] & [7] & [8] & [9] \\
\hline \multicolumn{10}{|c|}{ Panel C: Residual diagnostic test } \\
\hline$R^{2}$ & 0.920 & 0.893 & 0.964 & 0.952 & 0.808 & 0.815 & 0.177 & 0.851 & 0.865 \\
\hline$F_{\text {statistics }}^{2}$ & 9.496 & 13.691 & 16.633 & 10.753 & 11.487 & 9.496 & 13.691 & 16.633 & 7.223 \\
\hline$x_{\text {Auto }}^{2}$ & 0.113 & 0.672 & 0.375 & 0.270 & 0.057 & 0.690 & 0.716 & 0.979 & 0.981 \\
\hline$x_{\text {Hete }}^{2}$ & 0.720 & 0.637 & 0.926 & 0.882 & 0.782 & 0.331 & 0.107 & 0.317 & 0.112 \\
\hline$x_{N o r}^{2}$ & 0.992 & 0.437 & 0.023 & 0.554 & 0.476 & 0.532 & 0.423 & 0.466 & 0.887 \\
\hline$x_{R E S E T}^{2}$ & 0.116 & 0.717 & 0.955 & 0.085 & 0.022 & 0.631 & 0.912 & 0.292 & 0.193 \\
\hline
\end{tabular}

Note: $* * *, * *$, and $*$ indicate significance levels at $1 \%, 5 \%$, and $10 \%$, respectively.

Table 7. NARDL model estimation results

\begin{tabular}{|c|c|c|c|c|c|c|c|c|c|}
\hline & \multicolumn{3}{|c|}{ Private commercial bank } & \multicolumn{3}{|c|}{ State-owned commercial bank } & \multicolumn{3}{|c|}{ Foreign commercial bank } \\
\hline & [1] & [2] & [3] & [4] & [5] & [6] & [7] & [8] & [9] \\
\hline \multicolumn{10}{|c|}{ Panel A: Cointegration test } \\
\hline Fpass & 3.479 & 34.676 & 12.912 & 16.923 & 5.092 & $3.477^{*}$ & 11.823 & 12.901 & 6.091 \\
\hline Wpss & 12.345 & 12.457 & 11.054 & 7.084 & 11.041 & 7.041 & 10.447 & 16.754 & 10.447 \\
\hline$t D B M$ & -7.177 & -5.712 & -14.274 & -8.274 & & & & & \\
\hline \multicolumn{10}{|c|}{ Panel B: Long-run coefficients } \\
\hline$L R_{F I 1}^{+}$ & 0.095 & & & $0.061^{*}$ & & & $0.018 \mathrm{a}$ & & \\
\hline$L R_{F I 1}^{-}$ & $0.014 \mathrm{a}$ & & & $0.045_{a}$ & & & $0.069_{b}$ & & \\
\hline$L R_{F I 2}^{+}$ & & $0.021_{\mathrm{a}}$ & & & $0.078_{b}$ & & & $0.091_{b}$ & \\
\hline$L R_{F I 2}^{-}$ & & $0.069_{b}$ & & & $0.021_{b}$ & $0.084 \mathrm{a}$ & & 0.012 & \\
\hline$L R_{F I 3}^{+}$ & & & $0.112_{\mathrm{a}}$ & & & $0.071_{b}$ & & & $0.151_{b}$ \\
\hline$L R_{F 13}^{-}$ & & & $0.090_{b}$ & & & $0.068_{\mathrm{b}}$ & & & $0.092_{a}$ \\
\hline$L R_{F D I v o l}^{+}$ & $0.053_{\mathrm{a}}$ & $0.023_{b}$ & 0.018 & $0.089_{b}$ & 0.057 & $0.065_{b}$ & 0.019 & $0.011_{b}$ & $0.048_{b}$ \\
\hline$L R_{F D I v o l}^{-}$ & $0.073_{\mathrm{a}}$ & $0.042_{a}$ & 0.096 & $0.088_{a}$ & $0.049_{b}$ & $0.022_{a}$ & 0.064 & $0.047_{a}$ & $0.064_{a}$ \\
\hline$L R_{R}^{+}$ & $-0.092_{b}$ & $-0.0241_{9}$ & $-0.092_{a}$ & $-0.387_{b}$ & $-0.074_{b}$ & -0.069 & $-0.046_{b}$ & $-0.081_{b}$ & -0.045 \\
\hline$L R_{R}^{-}$ & $0.006_{b}$ & 0.022 & $0.006_{b}$ & $0.037_{b}$ & $0.033_{b}$ & $0.062_{b}$ & $0.094_{b}$ & $0.078_{b}$ & $-0.061_{b}$ \\
\hline$L R_{T O}^{+}$ & $-0.025 a$ & $-0.060_{a}$ & $-0.075_{b}$ & -0.074 & $-0.061_{b}$ & $-0.039_{b}$ & $-0.087_{b}$ & $-0.042_{b}$ & $-0.175_{b}$ \\
\hline$L R_{T O}^{-}$ & $-0.043_{b}$ & -0.085 & $-0.003_{b}$ & $-0.049 a$ & $-0.007_{b}$ & $-0.068_{b}$ & $-0.071_{b}$ & $-0.071_{b}$ & $-0.045_{b}$ \\
\hline \multicolumn{10}{|c|}{ Panel C: Short-run coefficients } \\
\hline$E C T-1$ & -0.389 & $-0.271_{a}$ & -0.260 & -0.401 & -0.389 & -0.207 & -0.263 & -0.175 & -0.220 \\
\hline$F I^{+}$ & $-0.012 b$ & - & - & $-0.012 b$ & - & - & $-0.018 b$ & - & - \\
\hline$F I^{-}$ & $-0.023 b$ & - & - & $-0.013 b$ & - & - & $-0.005 b$ & - & - \\
\hline $\mathrm{FI}^{+}$ & - & $-0.039 a$ & - & - & -0.024 & - & - & 0.016 & - \\
\hline$F I^{-}$ & - & $-0.009 a$ & - & - & -0.061 & - & - & -0.063 & - \\
\hline $\mathrm{FI}^{+}$ & - & - & -0.009 & - & - & -0.002 & - & -0.025 & -0.085 \\
\hline$F I^{-}$ & - & - & -0.008 & - & - & -0.004 & - & -0.017 & -0.016 \\
\hline$R^{+}$ & $-0.003_{a}$ & -0.002 & $-0.081_{a}$ & $-0.003_{\mathrm{a}}$ & 0.009 & -0.154 & -0.018 a & -0.065 & -0.037 \\
\hline$R^{-}$ & -0.029 & 0.004 & -0.002 & -0.002 & -0.377 & -0.099 & -0.021 & -0.040 & -0.024 \\
\hline $\mathrm{TO}^{+}$ & -0.007 & -0.024 & 0.003 & 0.011 & $-0.053_{\mathrm{a}}$ & -0.086 & $0.011_{b}$ & $-0.014_{b}$ & -0.003 \\
\hline$T O^{-}$ & -0.016 & $-0.015 a$ & -0.002 & -0.014 & -0.475 & $-0.048 a$ & -0.035 & -0.026 & $-0.004 b$ \\
\hline$F D I^{+}$ & -0.007 & -0.007 & 0.006 & -0.491 & -0.205 & -0.091 & 0.007 & -0.073 & 0.013 \\
\hline$F D I^{-}$ & -0.004 & $-0.006_{b}$ & -0.002 & -0.502 & 0.002 & 0.157 & -0.677 & -0.518 & $-0.004_{a}$ \\
\hline
\end{tabular}


Table 7 (cont.). NARDL model estimation results

\begin{tabular}{|c|c|c|c|c|c|c|c|c|c|}
\hline & \multicolumn{3}{|c|}{ Private commercial bank } & \multicolumn{3}{|c|}{ State-owned commercial bank } & \multicolumn{3}{|c|}{ Foreign commercial bank } \\
\hline & [1] & [2] & [3] & [4] & [5] & [6] & [7] & [8] & [9] \\
\hline \multicolumn{10}{|c|}{ Panel D: Symmetry test } \\
\hline$W_{L R_{-} F I 1}$ & 21.025 & - & - & $14.766_{\mathrm{a}}$ & - & - & $13.157 \mathrm{a}$ & - & - \\
\hline$W_{S R_{-} F I 1}$ & 18.148 & - & - & 11.628 & - & - & $22.814_{\mathrm{a}}$ & - & - \\
\hline$W_{L R_{-} F I 2}$ & - & $14.797_{\mathrm{a}}$ & - & - & $6.759_{c}$ & - & - & $11.277_{\mathrm{a}}$ & - \\
\hline$W_{S R_{-} F I 2}$ & - & $19.657 \mathrm{a}$ & - & - & 20.739 & - & - & $10.937 \mathrm{a}$ & - \\
\hline$W_{L R_{-} F I 3}$ & - & - & $15.202_{a}$ & - & & 8.317 & - & - & 12.398 \\
\hline$W_{S R_{F I 3}}$ & - & - & $9.762_{b}$ & - & - & $9.587_{b}$ & - & - & $13.938_{b}$ \\
\hline$W_{L R_{-} R}$ & $12.642_{a}$ & $11.628_{\mathrm{a}}$ & $13.603_{\mathrm{a}}$ & $11.964_{\mathrm{a}}$ & $12.384 a$ & $12.642_{\mathrm{a}}$ & $7.308_{c}$ & 15.266 & $13.788_{\mathrm{a}}$ \\
\hline$W_{S R_{-} R}$ & 5.823 & $7.946_{b}$ & 5.774 & 1.431 & $8.908_{\mathrm{b}}$ & 1.969 & 3.201 & 1.941 & 1.773 \\
\hline$W_{L R_{\_} T O}$ & $6.086_{c}$ & $6.051_{c}$ & $7.827 c$ & $6.826_{c}$ & $9.253_{b}$ & $14.086_{\mathrm{a}}$ & $14.305_{\mathrm{a}}$ & $14.655_{\mathrm{a}}$ & $10.302 a$ \\
\hline$W_{S R_{-} T O}$ & 4.328 & 1.867 & 0.071 & 2.527 & $9.034_{b}$ & 6.046 & $9.252_{b}$ & 5.944 & 1.78 \\
\hline$W_{L R_{2} F D I v o i}$ & $13.556_{b}$ & $8.891_{b}$ & $13.848_{a}$ & $6.742_{c}$ & $16.429 \mathrm{a}$ & 3.556 & 5.324 & $9.472_{b}$ & $14.4000_{\mathrm{a}}$ \\
\hline$W_{S R_{F D V v i}}$ & 2.585 & 1.659 & 4.139 & 8.14 & 2.788 & 9.384 & 6.775 & 3.786 & $9.31_{b}$ \\
\hline \multicolumn{10}{|c|}{ Panel E: Residual diagnostic test } \\
\hline$x_{\text {Auto }}^{2}$ & 0.614 & 0.886 & 0.387 & 0.330 & 0.928 & 0.219 & 0.620 & 0.384 & 0.681 \\
\hline$x_{\text {Hete }}^{2}$ & 0.708 & 0.295 & 0.052 & 0.562 & 0.235 & 0.728 & 0.748 & 0.420 & 0.903 \\
\hline$x_{\text {Normality }}^{2}$ & 0.632 & 0.187 & 0.933 & 0.119 & 0.017 & 0.292 & 0.162 & 0.480 & 0.137 \\
\hline$x_{R E S E T}^{2}$ & 0.924 & 0.290 & 0.481 & 0.619 & 0.570 & 0.821 & 0.794 & 0.609 & 0.233 \\
\hline
\end{tabular}

Note: The subscripts of $\mathrm{a} / \mathrm{b} / \mathrm{c}$ specify the level of significance at $1 \%, 5 \%$, and $10 \%$, respectively.

Table 8. Toda-Yamamoto causality test results ( $d m a x=4)$

\begin{tabular}{|c|c|c|c|c|c|c|c|}
\hline & $N P L$ & $F I$ & $F I$ & $F I$ & TO & $R$ & FDI_vol \\
\hline \multicolumn{8}{|c|}{ Panel A: For private commercial banks } \\
\hline$N P L$ & - & $11.889_{a}$ & 10.353 & $21.4399_{a}$ & $12.664_{a}$ & $21.853_{a}$ & 10.088 \\
\hline FI & 1.807 & - & 4.773 & 11.725 & 5.866 & $7.673_{b}$ & 0.761 \\
\hline$F I$ & $11.580_{\mathrm{a}}$ & $12.022_{\mathrm{a}}$ & - & 2.893 & 3.909 & 2.159 & $10.670_{a}$ \\
\hline FI & 3.586 & $10.533_{\mathrm{a}}$ & $7.443_{b}$ & - & 2.972 & 0.338 & 0.213 \\
\hline$T O$ & $8.438_{b}$ & $10.192_{a}$ & 1.107 & 0.446 & - & 0.310 & 1.393 \\
\hline$R$ & 14.299 & 3.682 & 5.348 & 10.948 & 14.669 & - & 10.129 \\
\hline$F D I$ vol & 2.286 & 3.468 & $10.383_{\mathrm{a}}$ & 2.569 & 3.204 & $13.047_{a}$ & - \\
\hline \multicolumn{8}{|c|}{ Panel B: For state-owned commercial banks } \\
\hline$N P L$ & - & $10.272 \mathrm{a}$ & $8.233 b$ & $15.149 a$ & $13.62 \mathrm{a}$ & $12.294 a$ & $14.078 \mathrm{a}$ \\
\hline FI & $12.754 a$ & - & 6.74 & $11.547 a$ & $10.972 a$ & $9.167 b$ & $11.364 a$ \\
\hline$F I$ & $10.75 a$ & $8.326 c$ & - & 2.316 & 4.76 & 2.937 & 0.067 \\
\hline$F I$ & $7.434 c$ & $11.553 a$ & 1.099 & - & 5.363 & 2.978 & 0.889 \\
\hline TO & $9.05 b$ & 1.241 & 0.465 & 1.475 & - & 5.54 & 1.097 \\
\hline$R$ & $11.132 a$ & 4.427 & $9.571 b$ & $17.839 a$ & 27.729 & - & 1.669 \\
\hline$F D I$ vol & $8.391 c$ & $12.931 a$ & 2.621 & 4.787 & 6.158 & $10.309 a$ & - \\
\hline
\end{tabular}


Table 8 (cont.). Toda-Yamamoto causality test results (dmax=4)

\begin{tabular}{|c|c|c|c|c|c|c|c|}
\hline & $N P L$ & $F I$ & $F I$ & $F I$ & TO & $R$ & FDI_vol \\
\hline \multicolumn{8}{|c|}{ Panel C: For foreign commercial banks } \\
\hline$N P L$ & - & $13.675 a$ & $17.613 a$ & $11.747 a$ & 4.039 & $10.052 a$ & $7.135 c$ \\
\hline$F I$ & 0.013 & - & 4.228 & 11.337 & 6.082 & 5.524 & 0.791 \\
\hline$F I$ & 10.671a & $9.933 b$ & - & $12.922 \mathrm{a}$ & 4.627 & 2.418 & 0.187 \\
\hline$F I$ & 0.354 & 0.315 & 0.445 & - & 0.115 & 4.193 & 3.02 \\
\hline TO & 5.324 & 0.161 & 1.189 & 0.861 & - & 1.827 & 0.267 \\
\hline$R$ & 2.974 & 1.337 & 4.428 & 1.19 & $9.207 b$ & - & 2.253 \\
\hline$F D I \_v o l$ & $21.87 a$ & 5.224 & $11.685 a$ & 2.183 & 10.152a & 1.21 & - \\
\hline
\end{tabular}

The next section deals with gauging the directional causality by performing equation (8), and the results are displayed in Table 8 . Causality results for NPL for private commercial banks are reported in a panel $\mathrm{A}$; results for state-owned bank are displayed in panel $\mathrm{B}$, and for foreign commercial banks - in panel C.

\section{DISCUSSION}

The results of the impact of financial innovation on NPLs establish a positive link between them. These findings indicate that the excessive financial innovation adaptation and diffusion adversely cause detreating the financial system's loan performance. Referring to the magnitudes of financial innovation, it is apparent that state-owned banks' loan performance in Bangladesh is more intense compared to other segments of bank operation. This finding advocates that bank-based financial institutions must behave rationally by understanding the significance of loan performance and possible consequences of excessive innovativeness in the credit portfolio.

FDI volatility and NPLs nexus reveal a positive association, indicating that the performance of FDI inflow variation in the economy determines the credit performance in the financial system. The evidence is that accumulating long-term capital for investment FDI is positioned on the top because FDI inflows accelerate domestic production activities and promote domestic enterprise expansion. Thus, any innovation in FDI, especially adverse shocks, directly injects a negative wave to- wards domestic firms, and their performance hinders FDI fluctuation. Eventually, the firm's loan performance to financial institutions hampered at large.

The role of remittances in Bangladesh's economy is well appreciated. In the case of overall loan performance $s$, remittance inflows enhance credit performance by decreasing the propensity to become a credit defaulter. The impact of remittance inflows on credit performance is positively associated, implying that remittances negatively cause NPLs in all segments of bank-based financial institutions. Moreover, in terms of remittance effects, private commercial banks are getting more assistance for decreasing the number of credit defaulters compared to state-owned and foreign commercial banks' operations in Bangladesh.

Trade openness augments aggregate output by expanding business activities by allowing them to participate in the international arena. Trade openness brings a positive wave into the economy, and financial institutions are not isolated from that wave. Referring to model findings, it is noticeable that trade openness helps financial institutions recover their loan performance, which ingresses that negative relationship between trade openness and NPLs.

The error correction term (ECT) represents the speed of adjustment towards long-run equilibrium, which is negative in sign and statistically significant. Table 6 shows that the error correction term (ECT-1) of each specified model is negative and statistically significant at a $1 \%$ significance 
level. The impact of financial innovation on NPLs is positive in most cases; however, only a few coefficients are statistically significant. Empirical models pass with several diagnostic tests, and their results are reported in Panel C of Table 6. Study findings reveal that all the empirically tested models are free from serial correlation, residuals of error are normally distributed, the issues of heteroskadacity are missing, and internal consistency and stability test confirms models capacity to produce unbiased estimation.

Asymmetric cointegration is evaluated by performing three tests: Fpss, Wpss, and tBDM, and their test statistics are displayed in Panel A (Table 7). All the test statistics are statistically significant at a $1 \%$ level, suggesting the rejection of the null hypothesis: "No asymmetric cointegration". This finding suggests that asymmetric cointegration prevails between financial innovation, remittance, trade openness, FDI volatility and NPLs.

In the long run, the asymmetric shocks that are positive and negative in financial innovation expose a positive linkage with NPLs. All the coefficients are statistically significant at a $1 \%$ or $5 \%$ significance levels. These findings suggest that the role of financial innovation is critical for the banks' credit performance. According to estimations, positive growth can intensify the current state of credit performance degradation and control in financial innovation to manage the present state of credit performance. Moreover, the magnitudes of positive innovation produce more intense than negative shocks in the financial system. Thus, the strategy formulation for credit expansion seeks special attention to gauge financial innovation's role, since it plays a meaningful role in financial development. The asymmetric shocks of FDI volatility establish a positive association with NPLs in the long run, and all the coefficients appear statistically significant at a $1 \%$ or $5 \%$ levels. A positive association suggests that stability in receiving FDI helps capital accumulation and aggregate productivity expansion, allowing growth in domestic firms. According to the coefficients of asymmetric shocks, i.e., positive and negative innovation, it is palpable that reducing the present state of credit situation can be improved by reducing FDI volatility, implying that stability in FDI inflows lessens the effects of volatility, which will bring positive wave in credit performance in the end. Positive shocks in remittance inflows establish negative associations with NPLs, and negative shocks in remittance inflows expose a positive association with NPLs. These findings suggest that continual inflows of remittances in the economy emerged as an alternative channel for money supply and support for capital formation.

Finally, the asymmetric effects of trade openness on NPLs reveal asymmetric shocks in trade openness and establish negative links with NPLs. Study findings suggest that domestic trade expansion allows firms' earning capacity and increases their credit payment capacity for credit approval from financial institutions. A negative tie between negative shocks and the state of NPLs reveals that decreasing the possibility of domestic trade expansion intensifies the deterioration of credit performance, indicating firms' incapacity for paying credit payment and becoming defaulter eventually.

The results of the short-run model estimation are displayed in Panel C of Table 7. The coefficient of the error term reveals a negative sign and is statistically significant at a $1 \%$ level. These findings suggest the speed of long-run convergence due to shocks in the short run, which refers to the shortrun asymmetric impact of financial innovation, remittances, trade openness, and FDI volatility on NPLs. Study findings establish a negative linkage between proxies of financial innovation and NPLs, but in terms of magnitudes, financial innovation is not as critical as appeared in the long run; however, all the coefficients are statistically significant. Regarding the remittance asymmetric shock, only positive variations expose a negative tie with NPLs, and all the coefficients are statistically significant at $1 \%$. Furthermore, the asymmetric impact of trade openness and FDI volatility establish statistically insignificant results in most cases.

Symmetry results for the long term are reported in panel $\mathrm{D}$ of Table 7 by performing the standard Wald test with the null hypothesis of symmetry. The Wald test statistics revealed the rejection of the null hypothesis and alternatively established asymmetric relations between financial innovation, remittances, trade openness, FDI volatility, and NPLs. Whereas in the short run, all three proxies of financial innovation establish an asym- 
metric association with NPLs. Besides, the Wald test results produce conclusive evidence supporting the presence of short-run asymmetry running between remittances, trade openness, FDI volatility and NPLs. The empirical model also undergoes several residual diagnostic tests and unveils estimation unbiasedness, efficiency, and stability when capturing the equation's probable effects.

The results of the study reveal quite a few causal associations between financial innovation, trade openness, remittances, FDI inflows, and NPLs. However, the study predominantly focuses on causality running towards non-performing loans. The study reveals a bidirectional causality between financial innovation and NPLs [FI $\leftrightarrow$ NPLs], trade openness and NPLs [TO $\leftrightarrow$ NPLs], as well as remittances and NPLs[R↔NPLs], which are available in Panel A and Panel B (Table 8). On the other hand, a bidirectional causality between FDI volatility and NPLs is established in Panels B and C. The findings suggest that domestic firm credit performance are largely dependent on macrofundamentals; hence, financial policymakers should emphasize the impact of macroeconomic movements on the implementation of financial policies.

\section{CONCLUSION}

The aim of this study is to gauge the effects of financial innovation, remittances, trade openness, and FDI volatility on credit performance in Bangladesh's financial system for the period 1981-2019. An empirical model estimation with AARDL reveals a positive association between financial innovation and NPLs in all banks in Bangladesh. Considering financial innovation elasticities on NPLs, it is evident that state-owned banks show more vulnerability in the process of detreating credit performance, compared to private and foreign banks in the financial system. This finding suggests that state-owned banks must make considerable efforts to understand the ultimate impact of financial innovation while deciding to adopt and disseminate financial innovation in their business models. Furthermore, a positive link reveals FDI volatility to NPLs, indicating that scarcity in capital adversely affects business operations, thus reducing the capacity to perform credit obligations to the financial institutions. This is because FDI inflows are critically significant for supplying capital and smooth business operations.

The results of non-linear ARDL test ascertain the presence of the asymmetric cointegration between financial innovation, remittances, trade openness, FDI volatility and NPLs. Moreover, financial innovation's asymmetric shocks expose a negative linkage with credit performance in all three model estimations. However, in terms of elasticities of asymmetric shocks, the study found that positive shocks in financial innovation are more prominent than negative variations. This fining explains that lessening the present state of credit performance, that is the reduction of credit defaults in the financial system, it is immensely significant to establish control over the speed of financial innovation diffusions in the system. The asymmetric effects of remittance and trade openness reveal a positive link with credit performance in the financial system. The role of remittances and trade openness is critically significant because of money supply management, financial efficiency, and capital accumulation from the expansion of domestic business. Finally, continual inflows of FDI are critical for the financial system, since longterm investment and capital asset mobilization increase aggregate productivity and create domestic enterprises' scope for glut earnings.

In summary, the findings reveal that excessive financial innovations play a detrimental role in bankbased financial institutions' credit performance, both in the long and short run. Thus, financial institutions have to be very cautious when offering innovative credit products, though financial innovation helps enhance financial performance; however, adverse effects can cause credit performance with a negative note. The expansion of domestic output accelerates the credit scope for the financial institutions and, at the same time, boosts credit performance. Therefore, contraction economic policies can adversely cause trade liberalization and, ultimately, contribute to credit de-regularization in the financial system. 


\section{LIMITATION AND FUTURE STUDY}

This form of research addresses the impact of macrofundamentals on credit performance and, to some extent, can produce one direction findings. An extended future study can be executed by considering key variables related to fiscal and monetary policy, since the credit performance of financial institutions, especially bank-based institutions, is highly dependent on government policy decisions. Furthermore, by taking other countries in the study, a comparative assessment can be made.

\section{AUTHOR CONTRIBUTIONS}

Conceptualization: Md. Qamruzzaman.

Data curation: Md. Qamruzzaman.

Formal analysis: Md. Qamruzzaman.

Funding acquisition: Md. Qamruzzaman.

Investigation: Md. Qamruzzaman.

Methodology: Md. Qamruzzaman.

Project administration: Md. Qamruzzaman.

Resources: Md. Qamruzzaman.

Software: Md. Qamruzzaman.

Supervision: Md. Qamruzzaman.

Validation: Md. Qamruzzaman.

Visualization: Md. Qamruzzaman.

Writing - original draft: Md. Qamruzzaman.

Writing - review \& editing: Md. Qamruzzaman.

\section{FUNDING INFORMATION}

This research work was funded by the Institute of Advanced Research (IAR), united international University (Grant Reference: UIU/IAR/01/2021/BE/07).

\section{REFERENCES}

1. Abid, L., Ouertani, M. N., \& Zouari-Ghorbel, S. (2014). Macroeconomic and bank-specific determinants of household's non-performing loans in Tunisia: A dynamic panel data. Procedia Economics and Finance, 13, 58-68. https://doi.org/10.1016/S22125671(14)00430-4

2. Adebola, S. S., Yusoff, W., \& Dahalan, J. (2011). An ARDL approach to the determinants of non-performing loans in Islamic banking system in Malaysia. Kuwait Chapter of Arabian Journal of Business and Management Review, 33(830), 1-11. Retrieved from https://www.arabianjbmr. com/pdfs/KD_VOL_1_2/3.pdf
3. Akinlo, O., \& Emmanuel, M. (2014). Determinants of nonperforming loans in Nigeria. Accounting \& Taxation, 6(2), 21. Retrieved from http://www. theibfr2.com/RePEc/ibf/acttax/atv6n2-2014/AT-V6N2-2014-3.pdf

4. Akter, R., \& Roy, J. K. (2017). The impacts of non-performing loan on profitability: An empirical study on banking sector of Dhaka stock exchange. International Journal of Economics and Finance, 9(3), 126-132. Retrieved from http://www.ccsenet.org/journal/ index.php/ijef/article/view/66419

5. Amin, A. S., Imam, M. O., \& Malik, M. (2019). Regulations, governance, and resolution of nonperforming loan: Evidence from an emerging economy. Emerging
Markets Finance and Trade, 55(10), 2275-2297. https://doi.org/10.1080 /1540496X.2018.1523788

6. Amuakwa-Mensah, F., \& BoakyeAdjei, A. (2015). Determinants of non-performing loans in Ghana banking industry. International Journal of Computational Economics and Econometrics, 5(1), 35-54. Retrieved from https:// www.researchgate.net/publication/262181229_Determinants_of_ non-performing_loans_in_Ghana_banking_industry

7. Anjom, W., \& Karim, A. M. (2016). Relationship between non-performing loans and macroeconomic factors with bank specific factors: a case study on loan portfolios-SAARC countries perspective. ELK Asia 
Pacific Journal of Finance and Risk Management, 7(2), 1-29. Retrieved from https://bhlss.files.wordpress. com/2017/02/2-relationshipbetween-non-performing-loansand-macroeconomic-factors.pdf

8. Banerjee, A., Dolado, J. J., Galbraith, J. W., \& Hendry, D. (1993). Co-integration, error correction, and the econometric analysis of non-stationary data. OUP Catalogue. Retrieved from https://oxford.universitypressscholarship.com/view/1 0.1093/0198288107.001.0001/ acprof-9780198288107

9. Beaton, K. M., \& Myrvoda, A. (2016). Non-Performing Loans in the Eccu. International Monetary Fund. Retrieved from https:// www.elibrary.imf.org/view/journals/001/2016/229/article-A001en.xml

10. Bhargava, A. (1986). On the theory of testing for unit roots in observed time series. The Review of Economic Studies, 53(3), 369384. Retrieved from https://www. jstor.org/stable/2297634? seq=1

11. Boudriga, A., Taktak, N. B., \& Jellouli, S. (2009). Banking supervision and non-performing loans: a cross-country analysis. Journal of Financial Economic Policy. https://doi. org/10.1108/17576380911050043

12. Chen, F.-W., Feng, Y., \& Wang, W. (2018). Impacts of financial inclusion on nonperforming loans of commercial banks: Evidence from China. Sustainability, 10(9), 3084.

13. Dickey, D. A., \& Fuller, W. A (1979). Distribution of the Estimators for Autoregressive Time Series with a Unit Root. Journal of the American Statistical Association, 74(366a), 427-431. https://doi.org/10.1080/01621459. 1979.10482531

14. Dimitrios, A., Helen, L., \& Mike, T. (2016). Determinants of nonperforming loans: Evidence from Euro-area countries. Finance Research Letters, 18, 116-119. Retrieved from https://ideas. repec.org/a/eee/finlet/v18y2016icp116-119.html
15. Dorina, C., \& Colesnicova, T. (2014). The impact of macroeconomic factors on nonperforming loans in the Republic of Moldova. Retrieved from https:// ideas.repec.org/a/vls/rojfme/ vly2014ilp73-78.htm

16. Elliott, G., Rothenberg, T. J., \& Stock, J. (1996). Efficient Tests for an Autoregressive Unit Root. Econometrica, 64(4), 813-836. Retrieved from https://www.jstor. org/stable/2171846? seq=1

17. Fofack, H. L. (2005). Nonperforming loans in Sub-Saharan Africa: causal analysis and macroeconomic implications. The World Bank. Retrieved from https://elibrary.worldbank.org/doi/ abs/10.1596/1813-9450-3769

18. Ghatak, S., \& Siddiki, J. U. (2001). The use of the ARDL approach in estimating virtual exchange rates in India. Journal of Applied Statistics, 28(5), 573-583. https://doi. org/10.1080/02664760120047906

19. Ghosh, A. (2015). Bankingindustry specific and regional economic determinants of nonperforming loans: Evidence from US states. Journal of Financial Stability, 20, 93-104. https://doi. org/10.1016/j.jfs.2015.08.004

20. Grigoli, F., Mansilla, M., \& Saldías, M. (2018). Macro-financial linkages and heterogeneous nonperforming loans projections: An application to Ecuador. Journal of Banking \& Finance, 97, 130-141. https://doi.org/10.1016/j.jbankfin.2018.09.023

21. Haniifah, N. (2015). Economic determinants of non-performing loans (NPLs) in Ugandan commercial banks. Taylor's Business Review, 5(2), 137-153. Retrieved from https://www. semanticscholar.org/paper/ Economic-Determinants-of-Nonperforming-Loans-in-Haniifah/40 021a0e11bb747d0270a50a9758db a00bfb51f8

22. Isik, O., \& Bolat, S. (2016) Determinants of non-performing loans of deposit banks in Turkey. Journal of Business, Economics and Finance, 5(4), 341-350. https:// doi.org/10.17261/Pressacademia.2017.356
23. Jiménez, G., \& Saurina, J. (2005). Credit cycles, credit risk, and prudential regulation. Bank of Spain. Retrieved from https:// www.ijcb.org/journal/ijcb06q2a3. htm

24. Kjosevski, J., \& Petkovski, M. (2017). Non-performing loans in Baltic States: determinants and macroeconomic effects. Baltic Journal of Economics, 17(1), 25-44. https://doi.org/10.1080/140609 9X.2016.1246234

25. Klein, N. (2013). Non-performing loans in CESEE: Determinants and impact on macroeconomic performance. International Monetary Fund. Retrieved from https://www.imf.org/en/Publications/WP/Issues/2016/12/31/ Non-Performing-Loans-in-CESEE-Determinants-and-Impacton-Macroeconomic-Performance- 40413

26. Kumar, R. R., Stauvermann, P. J., Patel, A., \& Prasad, S. S. (2018). Determinants of non-performing loans in banking sector in small developing island states: A study of Fiji. Accounting Research Journal, 31(2), 192-213. Retrieved from https://www.researchgate. net/publication/325180156_Determinants_of_non-performing_loans_in_banking_sector_in_small_developing_island_ states_A_study_of_Fiji

27. Kwiatkowski, D., Phillips, P., Schmidt, P., \& Shin, Y. (1992). Testing the null hypothesis of stationarity against the alternative of a unit root: How sure are we that economic time series have a unit root? Journal of Econometrics, 54(1-3), 159-178. Retrieved from https://doi.org/10.1016/03044076(92)90104-Y

28. Louzis, D. P., Vouldis, A. T., \& Metaxas, V. L. (2012) Macroeconomic and bankspecific determinants of nonperforming loans in Greece: A comparative study of mortgage, business and consumer loan portfolios. Journal of Banking \& Finance, 36(4), 1012 1027. Retrieved from https:// doi.org/10.1016/j.jbankfin.2011.10.012 
29. Louzis, D., Vouldis, A., \& Metaxas, V. (2010). Macroeconomic and bank-specific determinants of non-performing loans in Greece: A comparative study of mortgage, business and consumer loan portfolios. Journal of Banking \& Finance, 36(4), 1012-1027. Retrieved from https://doi.org/10.1016/j.jbankfin.2011.10.012

30. Makri, V., Tsagkanos, A., \& Bellas, A. (2014). Determinants of nonperforming loans: The case of Eurozone. Panoeconomicus, 61(2), 193-206. Retrieved from https:// ideas.repec.org/a/voj/journl/ v61y2014i2p193-206.html

31. McNown, R., Sam, C. Y., \& Goh, S. K. (2018). Bootstrapping the autoregressive distributed lag test for cointegration. Applied Economics, 50(13), 1509-1521. Retrieved from https://doi.org/10. 1080/00036846.2017.1366643

32. Minsky, H. P. (1964). Longer waves in financial relations: financial factors in the more severe depressions. The American Economic Review, 54(3), 324-335. Retrieved from https://digitalcommons.bard.edu/hm_archive/266/

33. Morakinyo, A. E., \& Sibanda, M. (2016). The Determinants of NonPerforming Loans in the MINT Economies. Journal of Economics and Behavioral Studies, 8(5), 39-55. Retrieved from https://ojs.amhinternational.com/index.php/jebs/ article/view/1430

34. Morakinyo, A., \& Sibanda, M. (2016). Non-performing loans and economic growth in Nigeria: a dynamic analysis. SPOUDAIJournal of Economics and Business, 66(4), 61-81. Retrieved from https://www.econstor.eu/handle/10419/169189

35. Ng, S., \& Perron, P. (2001). Lag length selection and the construction of unit root tests with good size and power. Econometrica, 69(6), 1519-1554. Retrieved from http://www.columbia.edu/ sn2294/pub/ecta01.pdf

36. Pesaran, M. H., Shin, Y., \& Smith, R. J. (2001). Bounds testing approaches to the analysis of level relationships. Journal of Applied
Econometrics, 16(3), 289-326. https://doi.org/10.1002/jae.616

37. Phillips, P. C. (1987). Time series regression with a unit root. Econometrica: Journal of the Econometric Society, 277-301. Retrieved from https://www.jstor. org/stable/1913237?seq=1

38. Phillips, P. C. B., \& Perron, P. (1988). Testing for a unit root in time series regression. Biometrika, 75(2), 335-346. Retrieved from https://www.jstor.org/ stable/2336182? seq $=1$

39. Polodoo, V., Seetanah, B., Sannassee, R., Seetah, K., \& Padachi, K. (2015). An econometric analysis regarding the path of non performing loans - a panel data analysis from Mauritian banks and implications for the banking industry. The Journal of Developing Areas, 49(1), 53-64. Retrieved from https:// ideas.repec.org/a/jda/journl/ vol.49year2015issuelpp53-64.html

40. Poudel, R. P. S. (2013).

Macroeconomic Determinants of credit risk in nepalese banking industry. Paper presented at the Proceedings of the 21st International Business Research Conference. Retrieved from https://nanopdf.com/download/ proceedings-of-21st-internationalbusiness-research-conference5b16f4e73db77_pdf

41. Qamruzzaman, M., \& Jianguo, W. (2017). Financial innovation and economic growth in Bangladesh. Financial Innovation, 3(1), 19. Retrieved from https:// jfin-swufe.springeropen.com/articles/10.1186/s40854-017-0070-0

42. Qamruzzaman, M., \& Jianguo, W. (2018). Does Foreign Direct Investment, Financial Innovation, and Trade Openness Coexist in the Development Process: Evidence from Selected Asian and African Countries? British Journal of Economics, Finance and Management Sciences, 16(1), 73-94 Retrieved from https://www.ajournal.co.uk/EFpdfs/EFvolume16(1)/ EFVol.16\%20(1)\%20Article\%207. pdf

43. Qamruzzaman, M., \& Jianguo, W. (2018). SME financing innovation and SME development in Bangladesh: an application of ARDL. Journal of Small Business \& Entrepreneurship, 1-25. Retrieved from https://doi.org/10.1080/0827 6331.2018.1468975

44. Qamruzzaman, M., \& Karim, S. (2020a). Do Remittance and Financial Innovation causes stock price through Financial Development: An Application of Nonlinear Framework. Retrieved from https://www.researchgate. net/publication/344693240_Do_ Remittance_and_Financial_Innovation_causes_stock_price_ through_Financial_Development_An_Application_of_Nonlinear_Framework

45. Qamruzzaman, M., \& Karim, S. (2020b). Nexus between Economic Voaltility, Trade Opennes and FDI: An Application of ARDL, NARDL and Asymmetric Causalty. Asian Economic and Financial Review, 10(7), 790-807. Retrieved from https://ideas.repec.org/a/asi/ aeafrj/2020p790-807.html

46. Qamruzzaman, M., \& Wei, J. (2018). Financial Innovation, Stock Market Development, and Economic Growth: An Application of ARDL Model. International Journal of Financial Studies, 6(3), 69. Retrieved from https://ideas.repec.org/a/gam/jijfss/v6y2018i3p69-d161634.html

47. Qamruzzaman, M., \& Wei, J. (2019). Financial Innovation and Financial Inclusion Nexus in South Asian Countries: Evidence from Symmetric and Asymmetric Panel Investigation. International Journal of Financial Studies, 7(4), 61. Retrieved from https://www. mdpi.com/2227-7072/7/4/6

48. Qamruzzaman, M., Bardhan, A., \& Nasya, S. (2020). Nexus between Remittance, Nonperforming Loan, Money Supply, and Financial Volatility: An Application of ARDL. International Journal of Applied Economics, Finance and Accounting, 8(1), 11 29. Retrieved from https:// econpapers.repec.org/article/ oapijaefa/2020_3ap_3a11-29.htm

49. Qamruzzaman, M., Jianguo, W., Jahan, S., \& Yingjun, Z. (2020). 
Financial innovation, human capital development, and economic growth of selected South Asian countries: An application of ARDL approach. International Journal of Finance \& Economics. Retrieved from https:// doi.org/10.1002/ijfe.2003

50. Qamruzzaman, M., Karim, S., \& Wei, J. (2019). Does Asymmetric Relation Exist between Exchange Rate and Foreign Direct Investment in Bangladesh? Evidence from Nonlinear ARDL Analysis. Journal of Asian Finance, Economics and Business, 6(4), 115-128. https://doi.org/10.13106/ jafeb.2019.vol6.no4.115

51. Rahman, B., \& Jahan, N. (2018). Non-Performing Loans (NPLs) in Islamic Banks of Bangladesh: An Empirical Study. Retrieved from https://islamicmarkets.com/ publications/non-performingloans-npls-in-islamic-banks-ofbangladesh-an-empirical

52. Rajha, K. S. (2016). Determinants of non-performing loans: Evidence from the Jordanian banking sector. Journal of Finance and Bank Management, 4(1), 125-136. Retrieved from http:// jfbmnet.com/journals/jfbm/ Vol_4_No_1_June_2016/9.pdf

53. Reinhart, C. M., \& Rogoff, K. S. (2011). From financial crash to debt crisis. American Economic Review, 101(5), 1676-1706. Retrieved from https://scholar. harvard.edu/files/rogoff/files/ from_financial_crash.pdf
54. Rifat, A. M. (2016). An Analytical Study of Determinants of NonPerforming Loans: Evidence from Non-Bank Financial Institutions (NBFIs) of Bangladesh. Journal of Business and Technology (Dhaka), 55-67. Retrieved from https:// www.banglajol.info/index.php/ JBT/article/view/34242

55. Rosenkranz, P., \& Lee, J. (2019). Non-performing Loans in Asia: Determinants and Macrofinancial Linkages. Asian Development Bank Economics Working Paper Series(574). https://dx.doi. org/10.22617/WPS190050-2

56. Sam, C. Y., McNown, R., \& Goh, S. K. (2019). An augmented autoregressive distributed lag bounds test for cointegration. Economic Modelling, 80, 130-141. https://doi.org/10.1016/j.econmod.2018.11.001

57. Schumpeter. (2021). The Theory of Economic Development. Cambridge: Harvard University Press. Retrieved from https://www.routledge.com/ The-Theory-of-EconomicDevelopment/Schumpeter/p/ book/9780367705268

58. Shin, Y., Yu, B., \& GreenwoodNimmo, M. (2014). Modelling asymmetric cointegration and dynamic multipliers in a nonlinear ARDL framework. In Festschrift in Honor of Peter Schmidt (pp. 281314). Springer. Retrieved from https://link.springer.com/chapter/10.1007/978-1-4899-8008-3_9

59. Shingjergji, A. (2013). The Impact of Macroeconomic Variables on the Non Performing Loans in the
Albanian Banking System During 2005-2012. Academic Journal of Interdisciplinary Studies, 2(9), 335. Retrieved from https://www.richtmann.org/journal/index.php/ajis/ article/view/855

60. Škarica, B. (2014). Determinants of non-performing loans in Central and Eastern European countries. Financial Theory and Practice, 38(1), 37-59. Retrieved from https://ideas.repec.org/a/ipf/finteo/ v38y2014ilp37-59.html

61. Stiglitz, J., \& Weiss, A. (1981). Credit Rationing In Markets With Imperfect Information. The American Economic, 85(1), 393-410. Retrieved from https://www.jstor. org/stable $/ 1802787$ ?seq $=1$

62. Tanasković, S., \& Jandrić, M. (2015). Macroeconomic and institutional determinants of non-performing loans. Journal of Central Banking Theory and Practice, 4(1), 47-62. Retrieved from https://ideas.repec.org/a/cbk/ journl/v4y2015ilp47-62.html

63. Toda, H. Y., \& Yamamoto, T. (1995). Statistical inference in vector autoregressions with possibly integrated processes. Journal of Econometrics, 66(1-2), 225-250. https://doi.org/10.1016/03044076(94)01616-8

64. Turan, G., \& Koskija, A. (2014). Non-performing loans in Albania. Academic Journal of Interdisciplinary Studies, 3(3), 491. Retrieved from https://www.richtmann.org/journal/index.php/ajis/ article/view/3451 